\title{
Elevated Plasmodium infection rates and high pyrethroid resistance in major malaria vectors in a forested area of Cameroon highlight challenges of malaria control
}

Cyrille Ndo ${ }^{1,2,3^{*}}$, Edmond Kopya ${ }^{1,4}$, Marie Agathe Donbou ${ }^{1,5}$, Flobert Njiokou ${ }^{4}$, Parfait Awono-Ambene ${ }^{1}$ and Charles Wondji ${ }^{2}$

\begin{abstract}
Background: High coverage of long-lasting insecticidal nets (LLINS) is the cornerstone of the malaria control strategy of the national malaria control program (NMCP) in Cameroon, with a target of reducing malaria transmission to less than $10 \%$ by 2035. To this end, more than 20 million LLINs have been distributed to populations countrywide since 2011. The present study evaluated entomological indices and Anopheles susceptibility to pyrethroids in a rural forested area of south Cameroon with high coverage of LLINs.

Methods: The study was conducted between July 2014 and May 2016 in Obout, a village located in a rural forested area in south Cameroon. Resting mosquitoes were collected using electric aspirators and were identified to species using morphological criteria and PCR tools. Mosquito feeding preferences and infection status to Plasmodium falciparum were determined by ELISA and using TaqMan assays. The susceptibility of wild F1 adults to pyrethroids was monitored using WHO insecticide susceptibility bioassays.

Results: During the study period, 5,993 Anopheles mosquitoes were collected indoors both in rooms with and without nets. Two main vector species, namely An. funestus and An. gambiae, were identified in the locality, with An. funestus being by far the most abundant (89.68\%). ELISA analysis revealed high percentage of blood meal taken exclusively on human (97.65-98.95\%) supporting the high antropohilic behaviour of both species. Plasmodium falciparum infection rate detected by ELISA was high throughout the study period and varied between 3.28-14.04\% (mean: 10.40\%) in An. funestus, and between 5.55-22.22\% (mean: 13.87\%) in An. gambiae. This trend was confirmed by TaqMan assays, with $P$. falciparum infection prevalence of $23.33 \%$ in An. funestus. Significant decrease of mortality associated with high frequency of kdr mutation was observed in An. gambiae (deltamethrin: 36.6-56.45\%; permethrin: 6-18.65\%) indicating high level of resistance to pyrethroids. For An. funestus, resistance was marked for deltamethrin (mortality: 70.54-76.24\%) than for permethrin (94.12-94.74\%).
\end{abstract}

Conclusions: Our study showed that despite LLINs, the population of Obout remains exposed to bites of highly infected An. funestus and An. gambiae mosquitoes, highlighting the challenges to controlling malaria in forested areas, especially in the presence of insecticide resistance.

Keywords: Malaria, Plasmodium falciparum, Pyrethroids, LLINs, Anopheles funestus, Anopheles gambiae, ELISA

\footnotetext{
* Correspondence: cyrndo@yahoo.fr

${ }^{1}$ Malaria Research Laboratory, Organisation de Coordination pour la lutte

Contre les Endémies en Afrique Centrale (OCEAC), P.O. Box 288, Yaoundé,

Cameroon

${ }^{2}$ Vector group, Liverpool School of Tropical Medicine, Pembroke Place,

Liverpool L3 5QA, UK

Full list of author information is available at the end of the article
} 


\section{Background}

The World Health Organization set the ambitious new target of reducing the global malaria burden by $90 \%$ by 2030 [1]. Vector control is the cornerstone of that strategy through the mass distribution of free long-lasting insecticidal nets (LLINs) [2, 3]. In Cameroon, the first national campaign of massive distribution of free LLINs was conducted in 2011 and increased the proportion of households possessing at least one LLIN from 33\% to $66 \%$. However, the universal coverage, defined as one LLIN to two people at risk of malaria [4], was achieved only at $32 \%$. Therefore, a second campaign was launched in 2015 with the aim to reach the $80 \%$ universal coverage targeted by the national malaria control program (NMCP) in the strategic plan 2011-2015 [5].

The universal coverage of LLINs has proved to be efficient in controlling malaria transmission in several subSaharan settings $[3,6]$. However, unexpected changes have been observed in Anopheles vector populations in some places following mass distribution campaigns of LLINs. For example, in An. funestus changes in biting behaviour were observed following massive introduction of LLINs in Benin and Senegal. This vector adopted early diurnal feeding and exophagic behaviour with the proportion of mosquitoes biting outdoor increasing to $26 \%[7,8]$. Moreover, a change in species composition in the An. gambiae complex after the implementation of LLINs was noticed in Dielmo (Senegal) [9]. In An. gambiae populations from the same area, Trape et al. [10] reported an increase in pyrethroid resistance characterized by rise of the frequency of Leu1014Phe $k d r$ resistance mutation from $8 \%$ in 2007 to $48 \%$ in 2010, after introduction of LLINs. Such changes could negatively impact malaria control operations by allowing mosquitoes to avoid contact or become resistant to insecticides.

As part of malaria control monitoring operations, it is necessary to regularly assess entomologic indices as well as level and mechanisms of insecticide resistance in vector populations in order to evaluate the effectiveness of control strategies implemented. Among the common indices recorded in vector populations are the species composition and abundance, entomological inoculation rate, blood-feeding preferences (antropophily/zoophily), biting (exophagy/endophagy) or resting (endophily/exophily) behaviour and Plasmodium infection rate [11, 12]. The present study aims to determine Anopheles species composition and abundance, anthropophily, Plasmodium infection rate and susceptibility profile to pyrethroids in a rural forested area of South Cameroon 3-5 years after mass distribution of LLINs.

\section{Methods}

\section{Study site and period}

The study started in July 2014, 3 years after the first national campaign of distribution of free LLINs, and ended in May 2016, two months after the end of the second campaign. It was conducted in Obout $\left(3^{\circ} 7^{\prime} \mathrm{N}, 11^{\circ} 65^{\prime} \mathrm{N}\right)$, a village located in a rural forested area close to the city of Mfou, situated about $25 \mathrm{~km}$ from Yaoundé, the capital city of Cameroon. The vegetation around the village is constituted by an equatorial forest which is degraded by farming activities. The climate is of equatorial guinean type, characterized by two rainy seasons (AugustOctober and April-June) and two dry seasons (NovemberApril and June-July). The annual average rainfall is $2000 \mathrm{~mm}$ while average annual temperatures range between $19-29{ }^{\circ} \mathrm{C}$, and the average humidity varies between $66-80 \%$ [13].

Obout is populated by about 200 inhabitants, most of whom are farmers. They live in houses made of mud or cement with tin rooves, presenting many interstices between the roof and the walls through which mosquitoes can enter or leave the houses. The village is also characterized by the presence of several fish ponds bordered with vegetation which could favour the development of immatures of Anopheles mosquito species, particularly those of $A n$. funestus group. The area is known to be hyperendemic for malaria $[13,14]$ and the main prevention method is LLINs, with coverage of around $70 \%$ in the population.

\section{Mosquito collection and morphological identification}

Resting mosquitoes were collected in human dwellings in the morning using electric aspirators (Rule In-Line Blowers, Model 240) and were brought back to the insectary. After species identification using morphological keys $[15,16]$, non-fed and some of the freshly blood engorged females were directly preserved in tubes containing desiccant for ELISA analysis to detect the presence of circumsporozoite protein (CSP) of P. falciparum in the head and thorax [17, 18], and to identify blood meal source [19]. The other blood-fed female mosquitoes were kept in paper cups for four days until eggs became mature. Gravid mosquitoes were allowed to oviposit according to the forced egg-laying [20], and eggs were reared to adult F1 used for insecticide bioassays, as described previously [21]. All the females were later killed and stored in tubes containing desiccant for future analysis.

\section{Laboratory processing of mosquitoes}

Dead mosquitoes stored in Eppendorf tubes containing desiccant were divided in several parts. Wings or legs were used for genomic DNA extraction as described previously [22] and morphological identification was confirmed using PCR based assays [23, 24]. The head and thorax were used for ELISA to detect $P$. falciparum CSP $[17,18]$ while abdomen containing blood was used for ELISA to identify the source of blood meal [25]. Diluted P. falciparum sporozoite proteins supplied by the Center 
for Disease Control (CDC, Atlanta, USA) were used as positive controls, while ground male mosquitoes were used as negative controls. For both ELISA analyses, optical densities (OD) were read at $405 \mathrm{~nm}$ on an ELISA plate reader (Biotek ELx800, Swindon, UK). Positive samples were determined by OD readings 2 -fold greater than the negative controls [17] and were tested a second time for validation.

Pattern of malaria transmission detected by ELISA was validated using TaqMan assay [26]. PCRs were done using the mosquito's whole body DNA extracts and the presence of $P$. falciparum $(\mathrm{F}+)$ and/or $P$. ovale, $P$. vivax and $P$. malariae $(\mathrm{OVM}+)$ was detected in 30 field-collected $A n$. funestus females. These females were randomly chosen and were different from those used for ELISA.

\section{Susceptibility assays to insecticides}

The susceptibility of wild F1 An. funestus and An. gambiae populations to discriminating concentrations of deltamethrin and permethrin was monitored using WHO insecticide susceptibility test-kits and standard procedures [27]. Impregnated papers were obtained from a WHO reference center (Vector Control Research Unit, University Sains Malaysia, Penang, Malaysia) and their quality was first checked on the susceptible Kisumu strain of An. gambiae. All tests were done at a temperature of $25-27^{\circ} \mathrm{C}$ and $80 \pm$ $10 \%$ relative humidity. For each test, four batches of 20-25 unfed F1 females, 2-5 day-old, were exposed to papers impregnated with an insecticide for $1 \mathrm{~h}$. Meanwhile, one batch of 20-25 mosquitoes exposed to untreated paper was used as a control. Percentage of knockdown (KD) mosquitoes was recorded at $60 \mathrm{~min}$, after which mosquitoes were held for $24 \mathrm{~h}$ at $27 \pm 2{ }^{\circ} \mathrm{C}$ and $80 \pm 10 \%$ relative humidity. The $\mathrm{kdt}_{50}$ and $\mathrm{kdt}_{95}$ which correspond to the time required for knocking 50\% and 95\% of mosquitoes tested, were estimated using a log-time probit mode [28]. Mortalities were recorded 24 h after exposure and were compared between the two years for each species and insecticide using Fisher's exact test run in Graph Pad prims V.5. P-values of $<0.05$ were considered as significant. Resistance/susceptible status was evaluated based on WHO criteria [27]. According to these criteria, mortality rates less than $90 \%$ were indicative of resistance while those greater than $98 \%$ were indicative of susceptibility. Mortality rates between 90-98\% suggested the possibility of resistance that needs to be confirmed. Finally, 50 F0 wild $A n$. gambiae mosquitoes were used for the detection of the L1014F and L1014S mutations by Taqmankdr assay [29].

\section{Results \\ Mosquito species composition}

A total of 5993 resting Anopheles mosquitoes were collected during the study period (Table 1). Repartition of mosquitoes according to the presence/absence or the quality of bednet showed that $71.12 \%$ were caught in rooms without mosquito net, $26.37 \%$ in rooms with old or perforated nets, while very few $(2.51 \%)$ were collected in rooms with new nets (no more than one year old). Two species groups/complexes, namely An. gambiae (s.l.) and An. funestus (s.l.), were identified according to morphological criteria, but the later was by far the most abundant representing $89.68 \%$ of the total mosquitoes caught. Molecular identification showed that An. funestus (s.s.) (hereafter $A n$. funestus) and An. leesoni where the two species of the funestus group present in Obout, with $A n$. funestus (s.s.) $(98.16 \%, n=213)$ being the most abundant. In the same manner, $A n$. gambiae $(93.96 \%, n=109)$ was much more abundant compared to An. coluzzii (3.45\%, $n=4)$; only three hybrids $(2.59 \%)$ between both species were identified.

\section{Blood meal source and Plasmodium circumsporozoite protein rate}

Overall, 95.56\% of mosquitoes were blood-fed, semi gravid or gravid at the time they were collected indicating that people living in surveyed houses were highly exposed to mosquito bites. ELISA analysis confirmed that An. funestus and An. gambiae were highly anthropophilic, with

Table 1 Number of An. funestus and An. gambiae mosquitoes collected in Obout (Cameroon), number tested by ELISA and corresponding circumsporozoite protein rates

\begin{tabular}{|c|c|c|c|c|c|c|c|c|c|}
\hline & & \multicolumn{3}{|l|}{2014} & \multirow{2}{*}{$\begin{array}{l}2015 \\
\text { Feb. }\end{array}$} & \multicolumn{4}{|l|}{2016} \\
\hline & & July & Oct. & All & & Feb. & Mar. & May & All \\
\hline \multirow[t]{4}{*}{ An. funestus } & Collected & 152 & 95 & 247 & 211 & 522 & 349 & 281 & 1152 \\
\hline & Tested & 152 & 95 & 247 & 211 & 522 & 349 & 281 & 1152 \\
\hline & Positive & 5 & 5 & 10 & 19 & 71 & 49 & 19 & 139 \\
\hline & ICSP (\%) & 3.29 & 5.26 & 4.04 & 9 & 13.6 & 14.04 & 6.76 & 12.07 \\
\hline \multirow[t]{4}{*}{ An. gambiae } & Collected & 36 & 9 & 45 & 174 & 160 & 85 & 84 & 329 \\
\hline & Tested & 36 & 9 & 45 & 174 & 160 & 85 & 84 & 329 \\
\hline & Positive & 2 & 2 & 4 & 17 & 28 & 13 & 14 & 55 \\
\hline & ICSP (\%) & 5.56 & 22.22 & 8.89 & 9.77 & 17.5 & 15.29 & 16.67 & 16.72 \\
\hline
\end{tabular}


97.65\% $(n=83 / 85)$ and $98.95 \%(n=94 / 95)$ blood meal exclusively taken on human, respectively, while less than $3 \%$ of tested samples consisted of mixed blood meals taken simultaneously on human and sheep.

Of the 2,170 head and thorax analyzed by ELISA, 245 were positive, corresponding to a high global circumsporozoite rate of $11.29 \%$. During the study period, this rate varied between $3.28-14.04 \%$ (mean: 10.4\%) in $A n$. funestus, and between 5.55-22.22\% (mean: 13.87\%) in An. gambiae (Table 1). For both species, the lowest infection rates were obtained in 2014, while the highest were obtained in 2016 in mosquitoes collected after the second campaign of distribution of free LLINs.

Using TaqMan assays, 8 mosquitoes of 30 tested were found to be infected by Plasmodium. Among these, 7 were infected with only P. falciparum $[\mathrm{F}+; 23.33 \%$ (7/30)] and 1 was infected with $P$. ovale, $P$. vivax and/or $P$. malariae [OVM+; 3.33\% (1/30)].

\section{Insecticide susceptibility}

A total of 1,336 An. funestus, 822 An. gambiae and 546 females from the $A n$. gambiae Kisumu strain were used in WHO susceptibility tests using two pyrethroids (permethrin and deltamethrin) (Table 2).

The An. gambiae Kisumu strain displayed fully susceptible phenotype for all insecticides tested with $\mathrm{KDT}_{50}$ less than $30 \mathrm{~min}$ and $100 \%$ mortality, indicating that the impregnated papers were of good quality. By contrast, high level of resistance to permethrin and deltamethrin, characterized by significant decrease of mortality coupled with increase in knockdown time, was observed in $A n$. gambiae. This resistance significantly increased one year to another [(Fisher's exact test for permethrin: $P<0.0001$; OR: 9.141; CI: 4.68-17.86 ); Fisher's exact test for deltamethrin: $P<0.0001$; OR: 5.124; CI: 3.82-8.00)] with mortality of $6 \%$ and $18.65 \%$ to permethrin and deltamethrin, respectively, in 2016 compared to $36.6 \%$ and $56.45 \%$, respectively, in 2015. (Table 2, Fig. 1). Using TaqMan-kdr assay, both L1014F and L1014S $k d r$ mutations were identified in An. gambiae population from Obout. However, the frequency of the L1014F (98.72\%) mutation was very high compared to that of the L1014S (7.95\%) mutation.

In $A n$. funestus, the level of resistance to deltamethrin was moderate, but also increased one year to another, with mortality rates of $76.24 \%$ and $70.54 \%$ in 2015 and 2016, respectively, but the difference was not significant [(Fisher's exact test: $P=0.283$; OR: 1.342 ; CI: $0.82-$ 2.19)]. For permethrin, low mortality rates (94.1294.74\%), suggesting probable resistance were recorded throughout the study period. Contrary to An. gambiae, the resistance to pyrethroid in An. funestus was not associated with elevation of $\mathrm{KDT}_{50}$, suggesting that $k d r$ is probably not involved in the resistance to pyrethroids in this species (Table 2, Fig. 1).

\section{Discussion}

The present study highlights important malaria transmission due to An. funestus and An. gambiae in a rural forested area of South Cameroon. Both species were present in the village throughout the year and were highly infected by $P$. falciparum, with infection rates reaching $22 \%$. However, the densities of An. funestus populations were significantly higher over the study period, making this species the major malaria vector in this locality, where it probably breeds in artificial fish ponds situated around the village.

Anopheles infection status was mainly determined by detecting the presence of $P$. falciparum circumsporozoite

Table 2 Mortality and knockdown time of An. funestus and An. gambiae from Obout (Cameroon) after 1 h exposure to pyrethroids

\begin{tabular}{|c|c|c|c|c|c|c|c|}
\hline Year & Species/strain & Insecticide & $n$ & $\mathrm{kdt}_{50}(\min )\left(\mathrm{IC}_{95}\right)$ & $\mathrm{kdt}_{95}(\min )\left(\mathrm{IC}_{95}\right)$ & Mortality (\%) & Status \\
\hline \multirow[t]{6}{*}{2015} & Kisumu & Perm. 0.75\% & 120 & $21.2(16.1-25)$ & $40(33.88-55.5)$ & 100 & S \\
\hline & & Delta. $0.05 \%$ & 100 & $18.1(17.6-18.9)$ & $24.9(23.4-27.4)$ & 100 & S \\
\hline & An. funestus & Perm. $0.75 \%$ & 152 & $17.2(16.8-17.7)$ & $22.4(21.4-23.8)$ & $94.74^{a}$ & PR \\
\hline & & Delta. $0.05 \%$ & 101 & $26.6(18.2-39.9)$ & $55.2(37.7-214.7)$ & $76.24^{b}$ & $\mathrm{R}$ \\
\hline & An. gambiae & Perm. 0.75\% & 153 & $>60$ & $>>60$ & $36.6^{c}$ & $\mathrm{R}$ \\
\hline & & Delta. 0.05\% & 124 & $38.3(35-41)$ & $>>60$ & $56.45^{\mathrm{e}}$ & $\mathrm{R}$ \\
\hline \multirow[t]{6}{*}{2016} & Kisumu & Perm. $0.75 \%$ & 167 & $21.12(16.1-24.6)$ & $40.3(33.88-55.5)$ & 100 & S \\
\hline & & Delta. $0.05 \%$ & 159 & $18(17.6-18.9)$ & $24.9(23.4-27.4)$ & 100 & S \\
\hline & An. funestus & Perm. 0.75\% & 510 & $29.41(22.52-38.4)$ & $41.61(32.15-52.86)$ & $94.12^{\mathrm{a}}$ & PR \\
\hline & & Delta. $0.05 \%$ & 573 & 39.62 (20.6-54.19) & 55.19 (44.89-90.19) & $70.54^{b}$ & R \\
\hline & An. gambiae & Perm. 0.75\% & 202 & $>60$ & $>>60$ & $6.00^{d}$ & R \\
\hline & & Delta. $0.05 \%$ & 343 & $>60$ & $>>60$ & $18.65^{f}$ & $\mathrm{R}$ \\
\hline
\end{tabular}

${ }^{a-f}$ For An. funestus and An. gambiae mortality rates for the same insecticide followed by different letters were significantly different between the two years (Fisher's exact test). All significant differences were at $P<0.0001$.

Abbreviations: kdt, Knockdown time; R, resistant; S, susceptible; PR, probably resistant; Perm., permethrin; Delta., deltamethrin. 


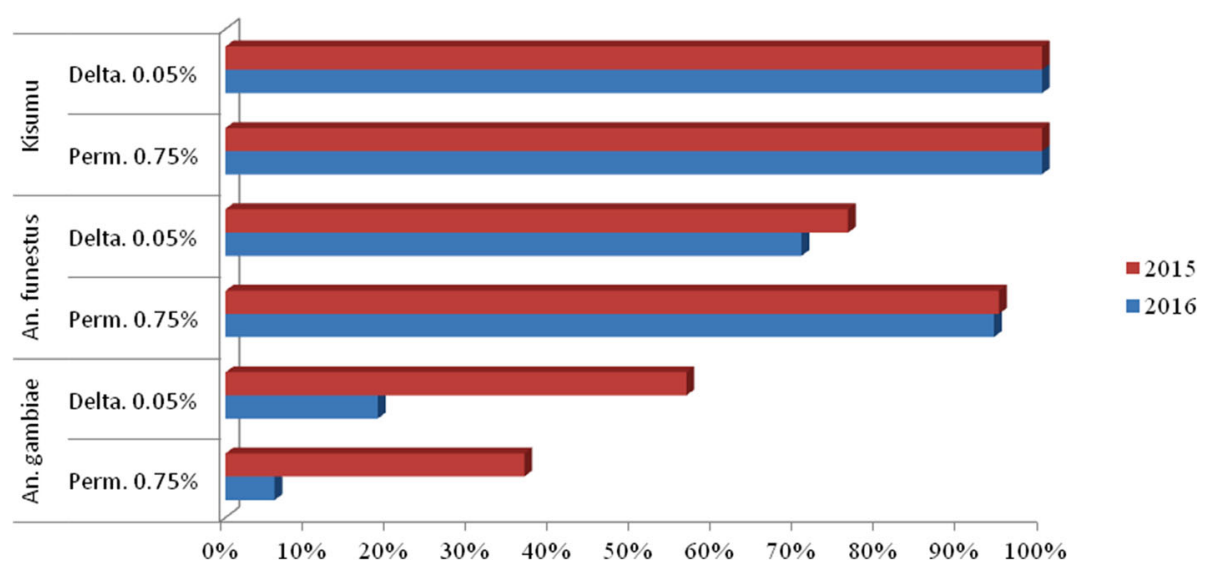

Fig. 1 Trends of mortality to permethrin and deltamethrin in An. funestus and An. gambiae mosquitoes from Obout (Cameroon) in 2015 and 2016

protein in the head and thorax of mosquitoes by ELISA. Although this technique could overestimate Plasmodium sporozoite prevalence by a factor of 1.1-1.9 in mosquitoes [30], the fact that TaqMan assays also detected high $P$. falciparum infection prevalence in An. funestus (2-fold than ELISA) reinforces the view that level of malaria transmission in the surveyed locality remains high, despite high coverage of LLINs.

LLIN represents the tool of choice for malaria control. It has significantly contributed to substantial reduction of malaria transmission in sub-Saharan African countries since its vulgarisation in $2000[3,6]$. LLIN plays a double role by protecting humans from mosquito bites and by killing mosquitoes which come in contact with the net. In this study, new mosquito nets received during the 2015-2016 national campaign of distribution of free LLINs were effective at preventing mosquito bites, since very few and non-fed mosquitoes were collected in rooms with new nets. In contrast, the fact that a non-negligible proportion (26.37\%) of blood-fed mosquitoes were collected in rooms with old LLINs received during the first national campaign of distribution of free LLINs in 2011 indicated that they could have lost their efficacy, probably due to several inappropriate washing or net deterioration. This highlights the need to replace LLINs that are torn or show waning efficacy to sustain high level coverage, in order to effectively reduce malaria transmission in sub-Saharan Africa [4, 30]. Regarding this, the WHO recommends that malaria endemic countries should supply LLINs through a combination of mass free distribution campaigns, normally at interval of no more than three years, and continuous distributions particularly during immunisation and antenatal services [31].

In addition, the high level of resistance to pyrethroids observed in malaria vectors in this study could have also contributed to the loss of net efficacy by allowing resistant mosquitoes to enter the nets or to bite through holes. Although our results showed that the $k d r$ L1014F mutation, and to lesser extend the L1014S mutation, were involved in the resistance observed in An. gambiae, it could not be biased to assume $k d r$ allele alone conferred the ability to survive diagnostic doses of pyrethroids. Thus, metabolic mechanisms [32, 33] must also contribute to the high-level of pyrethroid resistance in this species. This would also be the case for An. funestus, which exhibited moderate and probable resistance to delthamethrine and permethrin, respectively, owing to the fact that $k d r$ was never reported in this species. Similar to this study, difference in levels of insecticide resistance between sympatric populations of the two vector species was already reported elsewhere [34] and could be explained by difference in their biological characteristics. Anopeheles gambiae breeds in temporary stagnant waters, which are more polluted by agricultural pesticides, but less for An. funestus for which larvae develop in large semi-permanent water. Another main difference among the species could come from the fact that An. funestus probably recently colonized the surveyed locality, as fish ponds were less than three years old, and thus has not yet receive enough insecticide pressure selection. Further investigations are necessary to fully elucidate key insecticide resistance mechanisms for An. gambiae and An. funestus in our study area. Nevertheless, such a high level of resistance to the two pyrethroids used for net impregnation for major malaria vectors is a concern for the continued effectiveness of this key malaria control tool, and this call for an urgent development of new insecticide compounds with different mode of action [35].

Beside issues of insecticide resistance, partial coverage LLINs could also seriously limit the efficacy of malaria control operations in highly endemic settings such as forested areas of Cameroon. In fact, non-covered areas or houses without nets could represent hot spots of malaria transmission while people living in these places 
could serve as reservoir of Plasmodium parasites [36]. This hypothesis could also explain why a drop in malaria transmission (Anopheles infection) was not observed in the present study, even after the second campaign of distribution of LLINs. Our analysis therefore points out the necessity to increase LLINs coverage across Cameroon. and other control measures should be combined to LLINs in order to achieve the goal of reducing malaria cases to less than $10 \%$ by 2030 . These include indoor residual spraying (IRS), symptomatic diagnosis and treatment of malaria cases using artemisinin-based combination therapy (ACT) especially for children under 5 years, and prevention and control of malaria during pregnancy by administration of intermittent preventive treatment (IPTp) using Sulfadoxine-Pyrimethamine (SP). Moreover, since fish ponds represented major larval breeding sites in our studied area, perhaps combining larval control with LLINs should be considered.

\section{Conclusions}

The results of this study showed that the population of Obout sleeping in rooms without net or rooms with only old nets were highly exposed to bites of highly infected and pyrethroid resistant An. funestus and An. gambiae mosquitoes. In the context where malaria elimination is back again on the agenda of WHO and various stakeholders, the present study highlights the importance of achieving universal coverage of LLINs, the need to replace used LLINs two to three years after their distribution, and the necessity to implement additional malaria control measures in our study site. Meanwhile, more attention must be paid on the evolution of insecticide resistance in Anopheles vector species, which could seriously impede malaria control operations based on the use of insecticide or insecticide-treated tools including LLINs. Further studies are also necessary in order to investigate all factors which could explain such high level of malaria transmission despite large coverage of LLINs, by assessing human behaviour and use of LLINs, resting and biting behaviour of malaria vectors in the locality as well as mechanisms involved in insecticide resistance.

\section{Abbreviations \\ CSP: circumspororzoite protein; DDT: dichlorodiphenyltrichloroethane; ELISA: enzyme-linked-immunosorbent assay; IPTp: intermittent preventive treatment; IRS: indoor residual spraying; KD: knockdown; KDT: knockdown time; LLINs: long-lasting insecticidal nets; OD: optical density; PR: probably resistant; R: resistant; S: susceptible; SP: sulfadoxine-pyrimethamine; WHO: World Health Organization}

\section{Acknowledgements}

We are grateful to all the populations of Obout who allowed us to enter their houses for mosquito collection.

\section{Funding}

This work was supported by a Wellcome Trust Training Fellowship in Public Health and Tropical Medicine (102543/Z/13/Z) to CN. The Wellcome Trust had no role in the design of the study and collection, analysis, and interpretation of data and in writing the manuscript.

Availability of data and materials

All data generated or analysed during the current study are included in this published article.

\section{Authors' contributions}

Conceived the study and designed the study protocol: CN, CSW. Participated in field and laboratory analyses: CN, EK and MAD. CN analysed data and drafted the manuscript with contribution from CSW, PAA and FN. All authors read and approved the final manuscript.

Ethics approval and consent to participate

The study received the approval of the Cameroonian National Ethical Committee for Research on Human Health (statement No. 2015/01/535/CE/ NRERSH/SP). Non-written consent of household heads was sought prior to enter the house for mosquito collection.

Consent for publication

Not applicable.

\section{Competing interests}

The authors declare that they have no competing interests.

\section{Publisher's Note}

Springer Nature remains neutral with regard to jurisdictional claims in published maps and institutional affiliations.

\section{Author details}

${ }^{1}$ Malaria Research Laboratory, Organisation de Coordination pour la lutte Contre les Endémies en Afrique Centrale (OCEAC), P.O. Box 288, Yaoundé, Cameroon. ${ }^{2}$ Vector group, Liverpool School of Tropical Medicine, Pembroke Place, Liverpool L3 5QA, UK. ${ }^{3}$ Department of Biological Sciences, Faculty of Medicine and Pharmaceutical Sciences, University of Douala, P.O. Box 24157, Douala, Cameroon. ${ }^{4}$ Faculty of Sciences, University of Yaoundé I, P.O. Box 337, Yaoundé, Cameroon. ${ }^{5}$ School of Health Sciences, Catholic University of Central Africa, P.O. Box 11628, Yaoundé, Cameroon.

Received: 5 December 2017 Accepted: 28 February 2018

Published online: 08 March 2018

\section{References}

1. World Health Organization. Global Technical Strategy for Malaria 2016-2030. Geneva: World Health Organization; 2015.

2. Karunamoorthi K. Vector control: a cornerstone in the malaria elimination campaign. Clin Microbiol Infect. 2011;17:1608-16.

3. World Health Organization. World Malaria Report 2017. WHO Global Malaria Programme. http://www.who.int/malaria/publications/world-malaria-report2017/en/. Accessed 5 Jan 2018.

4. Zhou G, Li JS, Ototo EN, Atieli HE, Githeko AK, Yan G. Evaluation of universal coverage of insecticide-treated nets in western Kenya: field surveys. Malar J. 2014;13:351.

5. Ministry of Health of Cameroon. http://www.minsante.cm. Accessed $28 \mathrm{Apr}$ 2017.

6. Bhatt S, Weiss DJ, Cameron E, Bisanzio D, Mappin B, Dalrymple U, et al. The effect of malaria control on Plasmodium falciparum in Africa between 2000 and 2015. Nature. 2015:526:207-11.

7. Moiroux N, Gomez MB, Pennetier C, Elanga E, Djenontin A, Chandre F, et al. Changes in Anopheles funestus biting behavior following universal coverage of long-lasting insecticidal nets in Benin. J Infect Dis. 2012;206:1622-9.

8. Sougoufara S, Diedhiou SM, Doucoure S, Diagne N, Sembene PM, Harry M, et al. Biting by Anopheles funestus in broad daylight after use of long-lasting insecticidal nets: a new challenge to malaria elimination. Malar J. 2014;13:125.

9. Sougoufara S, Harry M, Doucoure S, Sembene PM, Sokhna C. Shift in species composition in the Anopheles gambiae complex after implementation of long-lasting insecticidal nets in Dielmo, Senegal. Med Vet Entomol. 2016;30:365-8

10. Trape JF, Tall A, Diagne N, Ndiath O, Ly AB, Faye J, et al. Malaria morbidity and pyrethroid resistance after the introduction of insecticide-treated bednets 
and artemisinin-based combination therapies: a longitudinal study. Lancet Infect Dis. 2011;11:925-32.

11. Gimnig JE, Vulule JM, Lo TQ, Kamau L, Kolczak MS, Phillips-Howard PA, et al. Impact of permethrin-treated bed nets on entomologic indices in an area of intense year-round malaria transmission. Am J Trop Med Hyg. 2003;68:16-22.

12. Mutuku FM, King CH, Mungai P. Mbogo C, Mwangangi J, Muchiri EM, et al. Impact of insecticide-treated bed nets on malaria transmission indices on the south coast of Kenya. Malar J. 2011;10:356.

13. Zeukeng F, Tchinda VH, Bigoga JD, Seumen CH, Ndzi ES, Abonweh G, et al. Co-infections of malaria and geohelminthiasis in two rural communities of Nkassomo and Vian in the Mfou health district, Cameroon. PLoS Negl Trop Dis. 2014;8:e3236.

14. Tchinda VHM, Ponka R, Ndzi ES, Madocgne AK, Amédée M, Tchinda MG, et al. Prevalence of malaria and soil-tranmitted helminth infections and their association with undernutrition in schoolchildren residing in Mfou health district in Cameroon. J Public Health Epidemiol. 2012;4:253-60.

15. Gillies MT, De Meillon B. The Anophelinae of Africa south of the Sahara. Publ Sth Afr Inst Med Res. 1968;54:1-343.

16. Gillies M, Coetzee M. A supplement to the Anophelinae of Africa South of the Sahara. Pub South Afr Inst Med Res. 1987;55:1-143.

17. Burkot TR, Williams JL, Schneider I. Identification of Plasmodium falciparuminfected mosquitoes by a double antibody enzyme-linked immunosorbent assay. Am J Trop Med Hyg. 1984;33:783-8.

18. Beier JC, Perkins PV, Koros JK, Onyango FK, Gargan TP, Wirtz RA, et al. Malaria sporozoite detection by dissection and ELISA to assess infectivity of afrotropical Anopheles (Diptera: Culicidae). J Med Entomol. 1990;27:377-84.

19. Beier MS, Schwartz IK, Beier JC, Perkins PV, Onyango F, Koros JK, et al. Identification of malaria species by ELISA in sporozoite and oocyst infected Anopheles from western Kenya. Am J Trop Med Hyg. 1988;39:323-7.

20. Morgan JC, Irving H, Okedi LM, Steven A, Wondji CS. Pyrethroid resistance in an Anopheles funestus population from Uganda. PLoS One. 2010;5:e11872

21. Ndo C, Kopya E, Menze-Djantio B, Toto JC, Awono-Ambene P, Lycett G, et al. High susceptibility of wild Anopheles funestus to infection with natural Plasmodium falciparum gametocytes using membrane feeding assays. Parasit Vectors. 2016;9:341.

22. Morlais I, Poncon N, Simard F, Cohuet A, Fontenille D. Intraspecific nucleotide variation in Anopheles gambiae: new insights into the biology of malaria vectors. Am J Trop Med Hyg. 2004;71:795-802.

23. Koekemoer LL, Kamau L, Hunt RH, Coetzee M. A cocktail polymerase chain reaction assay to identify members of the Anopheles funestus (Diptera: Culicidae) group. Am J Trop Med Hyg. 2002;66:804-11.

24. Santolamazza F, Mancini E, Simard F, Qi Y, Tu Z, della Torre A. Insertion polymorphisms of SINE200 retrotransposons within speciation islands of Anopheles gambiae molecular forms. Malar J. 2008;7:163.

25. Beier JC, Perkins PV, Wirtz RA, Koros J, Diggs D, Gargan TP, et al. Bloodmeal identification by direct enzyme-linked immunosorbent assay (ELISA), tested on Anopheles (Diptera: Culicidae) in Kenya. J Med Entomol. 1988;25:9-16.

26. Bass C, Nikou D, Blagborough AM, Vontas J, Sinden RE, Williamson MS, et al. PCR-based detection of Plasmodium in Anopheles mosquitoes: a comparison of a new high-throughput assay with existing methods. Malar J. 2008;7:177.

27. World Health Organization. Test procedures for insecticide resistance monitoring in malaria vector mosquitoes. Geneva: WHO; 2013.

28. Finney DJ. Probit analysis. 3. Cambridge, UK: Cambridge University Press; 1971.

29. Bass C, Nikou D, Donnelly MJ, Williamson MS, Ranson H, Ball A, et al. Detection of knockdown resistance (kdr) mutations in Anopheles gambiae: a comparison of two new high-throughput assays with existing methods. Malar J. 2007;6:111.

30. Fontenille D, Meunier JY, Nkondjio CA, Tchuinkam T. Use of circumsporozoite protein enzyme-linked immunosorbent assay compared with microscopic examination of salivary glands for calculation of malaria infectivity rates in mosquitoes (Diptera: Culicidae) from Cameroon. J Med Entomol. 2001;38:451-4.

31. World Health Organization. Achieving universal coverage with long-lasting insecticidal nets in malaria control. In: Global Malaria Programme. Geneva: WHO; 2014.

32. Corbel V, N'Guessan R, Brengues C, Chandre F, Djogbenou L, Martin T, et al. Multiple insecticide resistance mechanisms in Anopheles gambiae and Culex quinquefasciatus from Benin, West Africa. Acta Trop. 2007;101:207-16.
33. Ranson H, N'Guessan R, Lines J, Moiroux N, Nkuni Z, Corbel V. Pyrethroid resistance in African anopheline mosquitoes: what are the implications for malaria control? Trends Parasitol. 2011;27:91-8.

34. Kawada H, Dida GO, Ohashi K, Komagata O, Kasai S, Tomita T, et al. Multimodal pyrethroid resistance in malaria vectors, Anopheles gambiae s.s., Anopheles arabiensis, and Anopheles funestus s.s. in western Kenya. PLoS One. 2011;6:e22574.

35. Zaim M, Guillet P. Alternative insecticides: an urgent need. Trends Parasitol. 2002:18:161-3.

36. Bousema T, Griffin JT, Sauerwein RW, Smith DL, Churcher TS, Takken W, et al. Hitting hotspots: spatial targeting of malaria for control and elimination. PLoS Med. 2012;9:e1001165.

\section{Submit your next manuscript to BioMed Central and we will help you at every step:}

- We accept pre-submission inquiries

- Our selector tool helps you to find the most relevant journal

- We provide round the clock customer support

- Convenient online submission

- Thorough peer review

- Inclusion in PubMed and all major indexing services

- Maximum visibility for your research

Submit your manuscript at www.biomedcentral.com/submit
Biomed Central 